\title{
Research on Key Technology of TD-LTE Standard 4G Mobile Communi- cations Network
}

\author{
Dong QingXia ${ }^{1, *}$, Ding Gang ${ }^{2}$ and Chang HaiYan ${ }^{3}$
}

${ }^{I}$ School of Computer Science \& software, Tianjin Polytechnic University, Tianjin, 300387, P.R. China; ${ }^{2}$ Department of The Management and Construction of Teaching Resources Tianjin Radio \& TV University, 300191, Tianjin, P.R. China; ${ }^{3}$ Engineering Teach Practice Training Center, Tianjin Polytechnic University, Tianjin, 300387, P.R. China

\begin{abstract}
This paper first introduces the existing limitations of $3 \mathrm{G}$ network communication. It shows $4 \mathrm{G}$ is the inevitable trend of communication future development. $4 \mathrm{G}$ is a technology products which set $3 \mathrm{G}$ and WLAN in one, It has higher transmission speeds, greater capacity, better stability, may well make up for the deficiencies in $3 \mathrm{G}$. The article discusses and analyzes key technologies of $4 \mathrm{G}$ such as OFDM, software radio, smart antennas, MIMO and so on. It points out the improvements of $4 \mathrm{G}$ in technology and analyzes changes in $4 \mathrm{G}$ network architecture. Final it concludes the improvements of $4 \mathrm{G}$ network compared with $3 \mathrm{G}$. As the references of the new technology and the effectiveness improvement, this will bring $4 \mathrm{G}$ more broad application areas and market.
\end{abstract}

Keywords: OFDM, smart antenna technology, software defined radio, TD-LTE.

\section{INTRODUCTION}

The Third Generation (3G) mobile communication attracted much attention in the late 1980s but that in recent years has suffered cooling. The reason, from a technical point of view is, there are many areas should be improved for $3 \mathrm{G}$ systems, such as using circuit switching rather than pure IP way, just can provide the maximum rate only $384 \mathrm{kbit} / \mathrm{s}$ (maximum nominal rate of $2 \mathrm{Mbit} / \mathrm{s}$ ) which can not satisfy the requirements of the mobile communication system users, can't fully satisfy mobile video streaming media communication (demand) completely, without a global uniform standard, etc [1]. Because of shortages of $3 \mathrm{G}$, many people have come to expect $4 \mathrm{G}$ to solve these problems which can provide higher data rates, greater capacity and bandwidth.

\subsection{Problems in 3G Network}

Technology is Immature and the whole industry chain is not mature, resulting in both considerable problems still exist from the motor room, the base station to the mobile phone terminal.

Transmission speed is slow and instability. Theoretical peak speed of $384 \mathrm{k} / \mathrm{s}$, the current edge have reached a peak speed of $480 \mathrm{k} / \mathrm{s}$, but the average speed of its access to the Internet to download only about $10 \mathrm{~K}$.

Network coverage is not good. Because of its large base station equipment (single department station: about $2 \mathrm{~m}$, $500 \mathrm{~kg}-800 \mathrm{~kg}$ ), radiation seriously, that will give its full coverage of the network in the future laid significant obstacles.
Because TD network coverage can't be achieved seamlessly, there is no TD network where mobile phone users need to communicate via the GSM network. So now the TD mobile phones are used in the dual-mode GSM and TD structure. A big disadvantage of this structure is that the power consumption, which is a common problem with dualmode phones. Another fatal flaw is not a good solution TD mobile phone to GSM and GSM to TD network switching between crash problems.

Not international roaming. If the TD mobile phone was brought to Europe and America, simply can't use 3G. Global roaming $3 \mathrm{G}$ network is the most extensive WCDMA, including the more developed regions in Europe and America, Australia, Hong Kong, Macao, Taiwan, Japan, Korea, the Middle East, South America and Africa. India and Australia who have with CDMA all expressed its development of $3 \mathrm{G}$ is WCDMA.

It is because of the many deficiencies of $3 \mathrm{G}$, people have come to expect $4 \mathrm{G}$ to solve these problems, which can provide higher data rates, greater capacity and bandwidth. So make $4 \mathrm{G}$ closer personal communication than $3 \mathrm{G}$, technically better than the $3 \mathrm{G}$.

\subsection{G Communication}

$4 \mathrm{G}$ technology is the so-called fourth generation mobile communication technology and its abbreviation. It is a $3 \mathrm{G}$ and WLAN in one and be able to transmit high quality video images and image transmission quality comparable to highdefinition television technology products. Composition of $4 \mathrm{G}$ communication network is shown in Fig. (1). 4G communication speed theoretically achieves transmission of $100 \mathrm{Mbps}$. The bandwidth of $4 \mathrm{G}$ network is much higher than the bandwidth of $3 \mathrm{G}$ network cellular systems. Each $4 \mathrm{G}$ channel will occupy $100 \mathrm{MHz}$ spectrum, equivalent to 20 
times the W-CDMA 3G networks [2-3], and it can meet almost all users' requirements for wireless services. In addition, in the absence of cable modem or DSL coverage where 4G wireless networks can be deployed, further expansion throughout an area. 4G wireless network compared with the previous generation of $3 \mathrm{G}$ networks. $4 \mathrm{G}$ advantages are obvious and incomparable. Among all the communication quality and the speed of the data call are the most apparently.

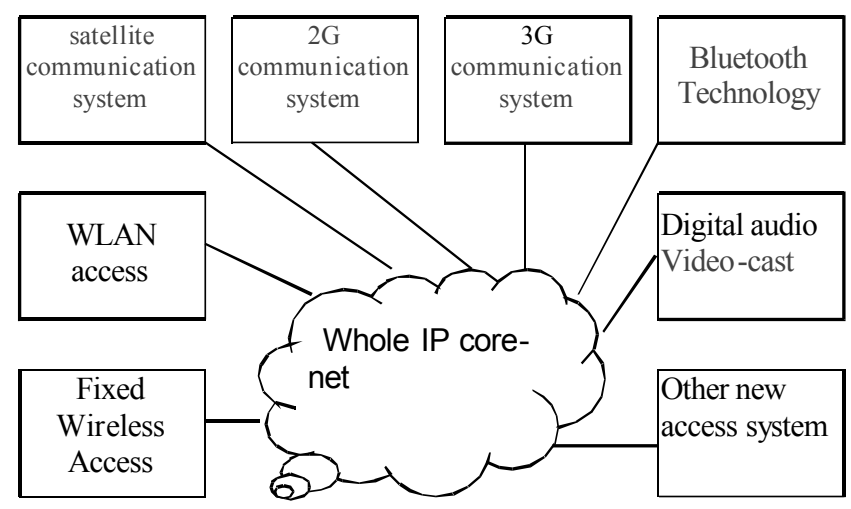

Fig. (1). Schematic diagram of $4 \mathrm{G}$ communication system network.

The $4 \mathrm{G}$ communication is not simply evolved from on the basis of $3 \mathrm{G}$ communications through a simple upgrade. Their core building technology is fundamentally different. the core technology of $3 \mathrm{G}$ mobile communication system is mainly CDMA, and 4G mobile communication system most attention technology is Orthogonal Frequency Division Multiplexing (OFDM).

$4 \mathrm{G}$ network has a trend of network convergence. In Fig. (1), the fixed wireless access, WLAN access, satellite communication system, GSM, 2G communication systems, 3G communication systems, Bluetooth access, digital audio and video broadcasting and other new access system will connect to whole-IP core network. "whole-IP core network" includes a whole from the IP transport layer to control layer and the application layer. Future wireless base station will have the ability to directly access to "Whole-IP core network" through IP protocol.

The main function of the existing $2 \mathrm{G}$ mobile communication such as system switching center MSC, the home location register HLR, authentication center AUC and other network elements etc will be achieved by server or database in $4 \mathrm{G}$ network, each layer of the protocol signaling online will be gradually replaced by IP protocol. The entire network will be evolved from the past Vertical tree type structure to distributed routing structure and business differences only reflect in access levels. The key technology in the 4G communication system that may be used include OFDM, software radio, smart antennas, MIMO, IP-based core network, etc. respectively. They are discussed below.

\subsection{TD-LTE}

The td- LTE is the abbreviation of TD-SCDMA Long Term Evolution, it is refers to the long-term evolution of tddma. The td-scdma LTE belongs to the second stage of the future evolution and development of td-scdma technology and standard connecting with TD- SCDMA and TDSCDMA enhanced technology standard.
LTE architecture (shown in Fig. (2)) make the 3GPP Release $6 \mathrm{RNC}$ and Node B for the integrated, it is eNB shown in Fig. (2). eNB provide E-UTRAN RLC / MAC / PDCP / function of physical layer protocol and control plane functions of RRC protocol, the entire system tends to flatten [4]. This change of the system structure and system makes interfaces of LTE reducing, the cost of equipment maintenance management reducing, become more convenient compared with UTRAN structures (shown in Fig. (3)), helping reduce the data transmission delay in terms of performance.

As an advanced technology, LTE requires the system to make further improve on increasing peak data rates and spectrum efficiency, and reducing the cost of network operation and construction. To enable the user to get "Always Online" experience, it need to reduce delay of TD-LTE control and user plane. The system must be able to coexist with existing systems $(2 \mathrm{G} / 2.5 \mathrm{G} / 3 \mathrm{G})$.

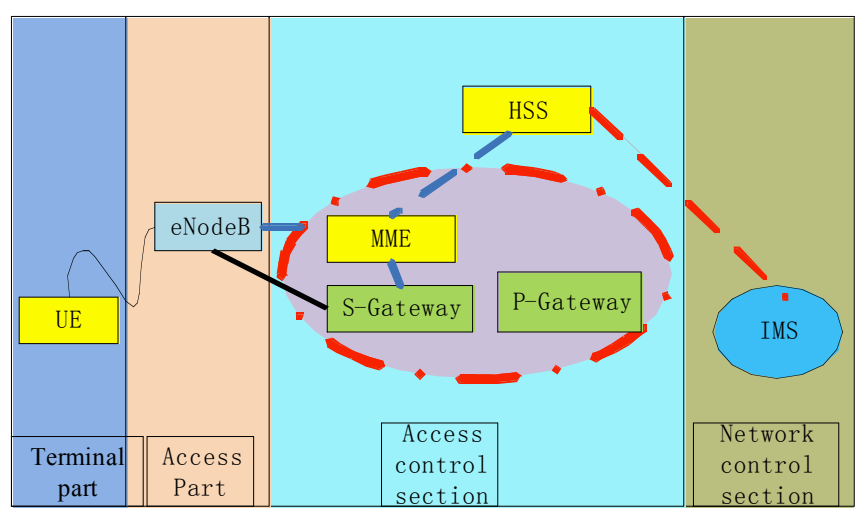

Fig. (2). LET Network architecture.

In the radio access network (RAN) side CDMA technology can be changed to OFDM (Orthogonal Frequency Division Modulation) technology which can against multipath interference in broadband systems more effective. OFDM technology has many advantages such as anti-multipath interference, simple and flexible to support different bandwidth and spectrum efficiency to support efficient adaptive scheduling etc. It is the $4 \mathrm{G}$ storage technology recognized. To further improve the spectrum efficiency, MIMO (multiple input / multiple output) technology has become a necessary LTE technology. By using spatial channel characteristics of multiple antenna system, MIMO technology can simultaneously transmit multiple data streams, so as to effectively increase the data rate and spectral efficiency.

To reduce the delay of the user plane and meet low latency (delay control surface is less than $100 \mathrm{~ms}$, the user plane latency less than 5ms) required, NodeB-RNC-CN structure must be simplified. RNC will cease to exist as a physical entity. NodeB RNC will have some features of RNC and become eNodeB. Network interconnection is made between eNodeB through the X2 interface and access to the $\mathrm{CN}$. Changes of system inevitably affect the network architecture changes. Changes of $4 \mathrm{G}$ network architecture is as follows.

Achieve a separation of control and bearer, MME is responsible for mobility management, signaling processing and other functions, S-GW is responsible for media stream processing and forwarding functions. 


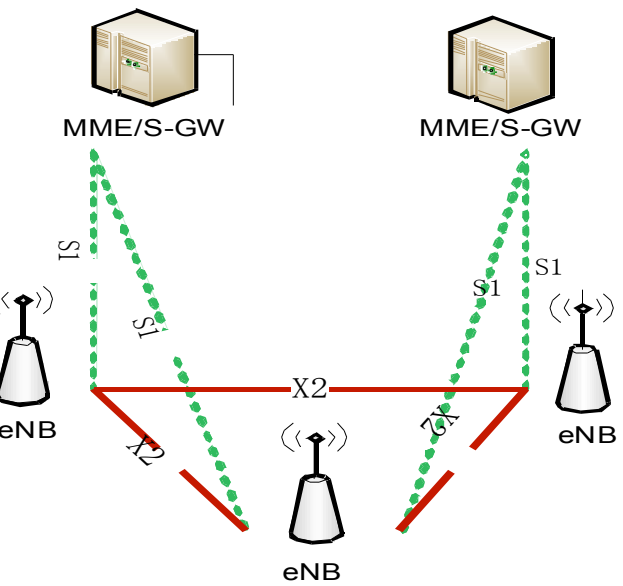

Fig. (3). E-UTRAN structure.

Core network canceled the CS (circuit domain). Whole IP's EPC (Evolved Packet Core) to support a variety of technical unified access, fixed-line and mobile convergence (FMC), Flexible support for VoIP and IMS-based multimedia services, the realization of whole IP network.

Canceled the RNC, RNC original function is distributed to the eNodeB and the gateway (GW), eNodeB direct access to the EPC, LTE network structure is more flat, so it reduces the user perceived latency and significantly enhance the user's mobile communication experience.

Introduce S1-Flex and X2 interfaces in Interface connection, Mobile hosts need to achieve multi-multipoint connections, $\mathrm{X} 2$ is the distributed interfaces between adjacent eNB, mainly for user mobility management. S1-Flex is the dynamic interface from the eNB to the EPC's, mainly used to improve network redundancy and load balancing.

\section{KEY TECHNOLOGIES IN 4G NETWORKS}

The TD-LTE introduces OFDM technology instead of CDMA in the basic multiple access technology, and introduces MIMO (Multiple-Input Multiple-Output) technology in the smart antenna (SA), further formed the SA + MIMO antenna technology more advanced. While the TD-LTE maintain original technical advantages and technical features such as specific time slot, synchronization and joint detection, It gets a huge promotion in the performance, but also keep smooth evolution of the td-scdma and enhancement tdscdma network to TD-LTE networks.

\subsection{Multiple Access Physical Layer}

The physical layer is the foundation for a wireless communication system. LTE downlink adopt orthogonal frequency division multiple access OFDMA, uplink using single carrier frequency division multiple access (SC-FDMA) for multiple access.

OFDM (Orthogonal Frequency Division Multiplexing) is a high-speed transmission technology in wireless environment, the basic principle of this technique is to transform the high-speed serial data into a plurality of relatively low-speed parallel data and modulate the different carrier. This parallel transmission system greatly expands the pulse width of the symbol and improves the performance of anti multipath fad- ing. Modern OFDM system uses digital signal processing techniques, generation and reception of each sub carrier is implemented by digital signal processing algorithm, this greatly simplifying the system structure. Meanwhile, in order to improve spectral efficiency and make each sub-carrier spectral overlap (shown in Fig. (4)), But these spectral should be orthogonality in the symbol period to ensure that the receiving side can recover the signal without distortion.

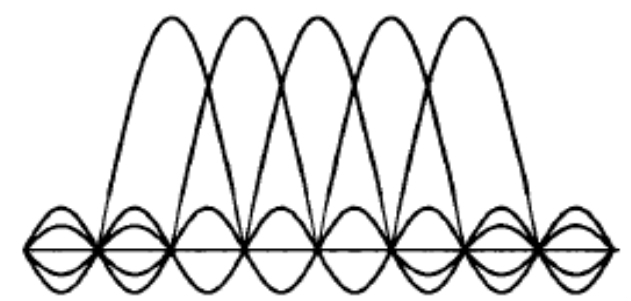

Fig. (4). Schematic view of an orthogonal frequency division multiplexed signal spectrum.

OFDM is a key feature of the LTE system. It is the core technology of the fourth generation of mobile communication. OFDM's basic idea is distributed the high speed data stream on a plurality of orthogonal subcarriers to be transmitted, so that the symbol rate of the sub-carriers is greatly reduced but symbol duration become much longer, thus the system can have a strong resistance to delay spread and the effects of inter-symbol interference is reduced. Usually the guard interval symbols is added before OFDM symbol, as long as the guard interval is greater than the channel delay spread inter-symbol interference ISI can completely be eliminate.

OFDM parameter settings will have a decisive impact on the performance of the entire system, such as a cyclic prefix. It is mainly used to effectively eliminate inter-symbol interference, and its length determines the anti-multipath capacity of the OFDM system and the transmission cover ability. In order to achieve the coverage requirements of the cell radius $100 \mathrm{~km}$, LTE system using two cyclic prefix length. The long $\mathrm{CP}$ help to overcome multipath interference and support covering a wide range, however, the overhead will also increase, resulting in data transfer capability decreases. Short $\mathrm{Cp}$ program is the basic option. Long $\mathrm{CP}$ program use to support LTE systems covering a wide range of services and multi-cell broadcast.

On upstream direction, LTE system uses SC-FDMA technique based with cyclic prefix. The biggest advantage is to reduce the transmitting terminal peak to average power ratio and reduce the size and cost of the terminal. Its features also include flexible spectrum bandwidth allocation, a fixed sequence of subcarriers, cyclic prefix against multipath fading and the variable transmission time interval.

LTE carrier spacing is one of the basic parameters of the system. Through theoretical analysis and simulation, ultimately determine the use of $15 \mathrm{k} \mathrm{Hz}$. The minimum uplink and downlink resource blocks are $375 \mathrm{k} \mathrm{Hz}$, the width is 25 subcarriers. The manner of mapping data to the resource block can be a centralized or discrete. Through the rational allocation of the number of subcarriers, the system can achieve $1.25 \sim 20 \mathrm{MHz}$ flexible bandwidth allocation. 


\subsection{Software Defined Radio}

Software Defined Radio can contact different forms of communication technologies in together. The basic idea of Software Defined Radio is to digital analog signal as closer as possible the antenna, That is to making the $\mathrm{A} / \mathrm{D}$ and $\mathrm{D} /$ A converter as closer to the RF front-end, using the DSP channel separation techniques, modulation and demodulation and channel encoding and decoding, etc. By establishing a highly flexible software and hardware platform in which various software can run, SDR can realize the wireless communication of multi-channel, multi-level and multimode, and realize the communication between different systems and platforms, and thus achieve "Without Borders Internet "technology platform world.

An ideal Software Defined Radio composition structure is shown in Fig. (5) Software Defined Radio is mainly composed of an antenna, RF front-end, broadband A / D and D / A converters, general and specialized digital signal processors and visual software. The antennas in Software Defined Radio generally cover a relatively wide frequency band, characteristics of each band is uniform in order to meet the needs of various businesses. RF front-end completed frequency conversion, filter, power-amplifier and other tasks in the launch. When receiving to realize filtering, amplification, frequency conversion and other functions. The analog signal processing tasks digitized full commitment by the DSP software. In order to lessen the pressure of the common DSP, typically the digital signal transmitted by the A / D converter, first processed by a special digital signal processor to reduce the data flow rate, then changed to the baseband signal, finally the data sent to general-purpose DSP processing.

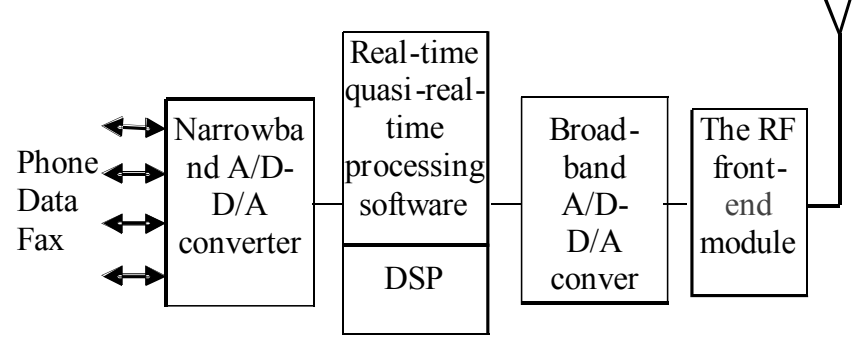

Fig. (5). Software defined radio block diagram.

Because a variety of mobile communication standards join, the existing mobile communication standard family has become very complicated. For the recent development, software defined radio technology can solve the compatibility problem of different standards and make global roaming convenient. For the long-term development, the goal of software defined radio development is to realize a intelligent wireless communication system which can adaptively configure receiver/ transmitter data rate, modulation, demodulation, channel coding and decoding methods, and even adjust the channel frequency, bandwidth and wireless access technology according to the radio to environmental changes, thereby making better use of spectrum resources to meet the requirements of the user on the basis of the maximum system capacity. I believe that as SDR technology continues to mature and develop, its role in $4 \mathrm{G}$ will become increasingly prominent, which will accelerate the improvement of $4 \mathrm{G}$ systems.

\subsection{Smart Antenna Technology}

Smart Antenna (Smart Antenna, SA) is also called an adaptive array antenna. It is composed of antenna array, beam forming network, adaptive algorithm three parts (shown in Fig. (6)). SA algorithm adjust the amplitude and phase weighting of each element signal by satisfying certain criteria, and adjust the shape of the antenna array pattern to enhance the desired signal of the interference signal suppression. SA can inhibit signal interference, automatic tracking and smart features such as digital beam adjustment, and it is considered to solve the shortage of frequency resources, effectively enhance the system capacity, and improve the transmission rate of communication pathways to ensure effective communication quality.

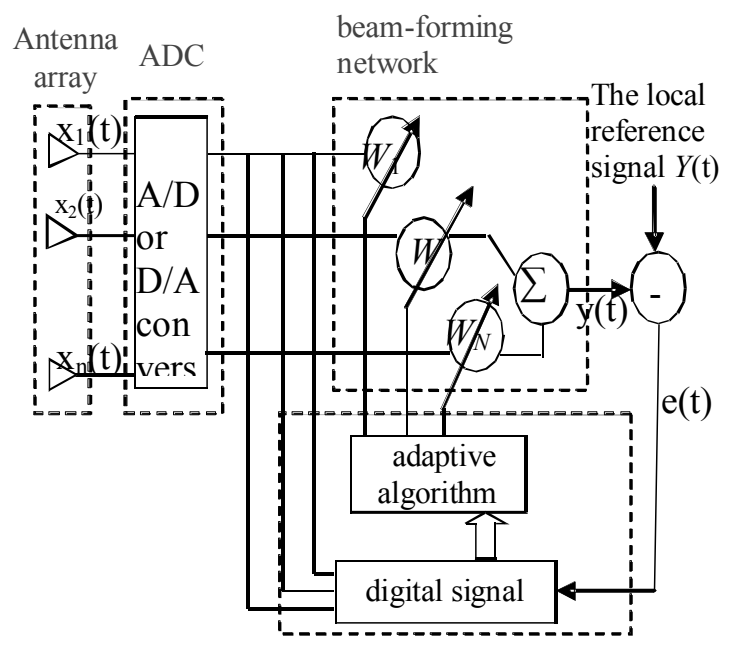

Fig. (6). Smart antenna structure diagram.

Principles of smart antenna is the antenna main beam aimed at the target user signal arrive direction, commit sidelobe or zero on interfering signals arrive direction, to achieve full and efficient use of the mobile user signal and delete or inhibit interfering signal purposes. Meanwhile, because of differences between the various spatial signature of mobile users smart antenna technology can receive and transmit a plurality of mobile users signals on the same channel without interfering with each other by using array antenna technology, so to make the use of the radio spectrum and the signal transmission is more efficient [5]. Without increasing system complexity, the use of smart antennas can meet quality of service and network expansion needs.

Intelligent algorithm is the core of the smart antenna system. Intelligent algorithm determines the instantaneous response rate and the complexity of the circuits, so it is important to select a good algorithm to achieve intelligent control beam. Algorithm automatically adjusts the weighted worthy to get the role of space required and the frequency filter.

\subsection{MIMO Technology}

Multi antenna technology (shown in Fig. (7)) refers to the use of the downlink MIMO (Multiple Input Multiple Output) and transmitting diversity technique. LTE multi-antenna technology basic configuration is a $2 \times 2$ antenna of doubleemission and double-receiving configuration in downlink, in uplink uses $1 * 2$ antenna configuration of single- emission 
double-receiving. Highest requirements at this stage to consider is the downlink MIMO and antenna diversity support four transmit four received $4 * 4$ antenna configurations or four transmit double received $4 * 2$ antenna configuration [6].

MIMO technology sets multiple transmission antennas and multiple receive antennas on the transmitter and the receiver, by the combination of multiple transmission antennas and multiple receive antennas to improve the communication quality for each user, or to improve the communication efficiency. MIMO channel can increase the radio channel capacity exponentially without increasing the bandwidth and the antenna transmitting power, the spectral efficiency can be improved exponentially.

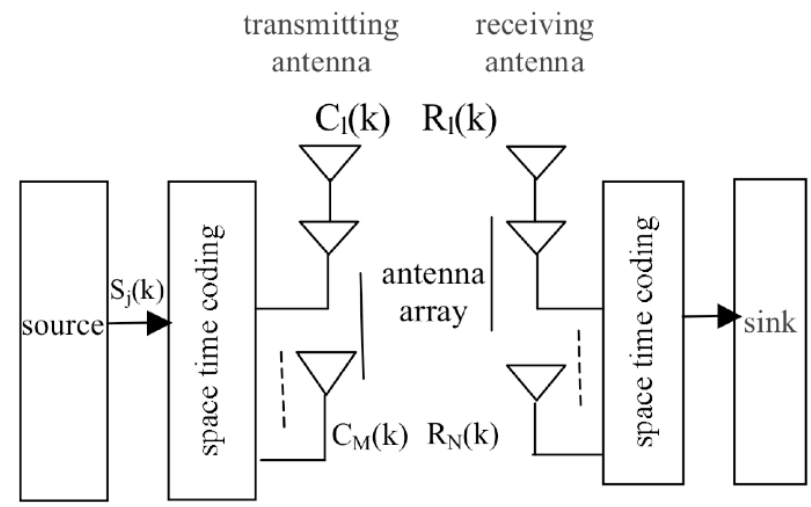

Fig. (7). Multi antenna.

Transmitting and receiving multi-antenna system is an important part of the MIMO wireless system. Its performance directly affects the performance of the MIMO channel. Signal emitted by multi-antenna is scattering propagation and mixed together in the wireless channel, after received by a multi-antenna, system through space-time processing algorithm to separate and recover the transmitted data, its performance depend on the independence level of every antenna unit receiving the signal, i.e. correlation, and the correlation between the antennas is more closely related to the antenna characteristics and the scattering propagation. Therefore, MIMO system high performance not only depends on Richness of multipath propagation, but also on more rational design of the antenna unit.

MIMO technology is essentially to provide the spatial multiplexing gain and diversity gain for the system [7]. Spatial multiplexing to increase the channel capacity, spatial diversity is to improve the reliability of the channel and reduce the bit error rate of the channel. The key of MIMO technology is to change the Influence factors of multipath fading in the presence of a conventional communication system into communication performance advantage enhancement factor to user. Effective use of random fading and multipath propagation may existing to exponentially increase the transmission rate of the business, Therefore it is possible to improve the performance of wireless communication of several orders of magnitude without increasing the occupied bandwidth of the signal provided.

\section{APPLICATION OF 4G NETWORK}

4G can transmit information, pictures, video voice using two-way while a voice call. $4 \mathrm{G}$ phones can timely remind the phone's owner what to do or should not do at this time based on environmental factors, time and other settings of factors. You can check road conditions on the phone through live video and select a nearest and vehicle at least way to work. You can also shop online eliminating the need to go to a crowded supermarket trouble. You can directly purchase tickets and tickets according to your own needs on $4 \mathrm{G}$ phones.

\subsection{G Application in Smart Phone}

Use either SI (MKS) or CGS as primary units. (SI units are encouraged.) If your native language is not English, try to get a native English-speaking colleague to proofread your paper. Do not add page numbers.

\subsection{G Applications in the Smart Home}

$4 \mathrm{G}$ would be a super-high-speed wireless network, a information superhighway which does not require cable. This new network enables phone users establish a connection by wireless and three virtual reality space. $4 \mathrm{G}$ communication allows more fluid communication between people, particularly the construction of a free communication channel between man and machine. Based on $4 \mathrm{G}$ mobile technology, "smart home" will combine wired and wireless to construct advanced wired and wireless network, Each type of product will have become Internet terminals. These terminals have IntelliSense, interconnection and coordination sharing feature, you can achieve communication between people, people and home appliances, even home appliances and appliances.

The era of smart appliances in the network will provide the best wisdom life experience to the user, and to build "a cloud of N-terminal" infrastructure.

\subsection{G Applications of Live TV}

3G/4G way has many advantages, such as low cost, easy to use, long distance transmission, low equipment cost, existing large scale network build by operator, less blind urban area. So television media breaking news live (transmission) or live (transmission) in transportation, power supply conditions harsh terrain of places, using $3 \mathrm{G} / 4 \mathrm{G}$ way is a very good choice.

\subsection{G Applications in the Field of Security}

The network monitoring has become a major trend in security industry environment. Currently Monitoring trending HD is becoming increasingly obvious. High-definition monitor widely used in front-end make the pressure transmission of back-end becoming heavier. 4G network introduce its mobile wireless transmission advantage in mobile surveillance, to bring a better environment for the development of network video surveillance (shown in Fig. (8)).

The amount of data generated by security products program becomes more and more, user demands for a longer storage time and a faster storage speed. For Video monitoring equipment cloud technology will significantly expand its storage space and reduce its storage costs. Because there is no support of fairish network connection technical, Cloud technical is lack of stability and data is difficile to store. Our 
security companies want to develop cloud storage, the first important is to be supported by the bandwidth and wireless network speed and stability.

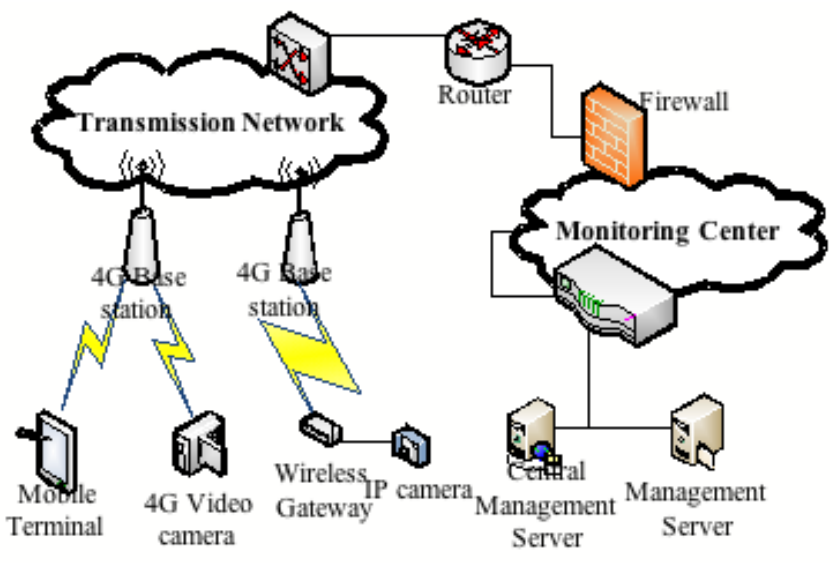

Fig. (8). Mobile monitoring system.

Therefore, the development of the 4G technology has a major role in promoting for entire security industry. 4G technology will enable the integration of cloud technologies and security technologies. It not only give full play to the technological advantages of the security industry, but also take full advantage of cloud technology to bring convenient and affordable storage.

\subsection{Other Applications}

$4 \mathrm{G}$ network can provide better, faster and cheaper health care and emergency services, and in the fight against floods, earthquakes it play an important role.

\section{DEVELOPMENT OF 4G TERMINALS}

\subsection{Handheld Hardware Development}

In the future mobile phone has a higher pixel camera, people can always make things around into a video uploaded to share with friends online. They need not worry about network congestion caused by a large number of users to use the network even higher frequencies. If AR (Augmented Reality Technique) is in combination with traditional life business, users can make the combination of virtual and reality by mobile phone.

For a scene example, when a man make an appointments with a friend in a strange place, by AR combines GPS technology and video phone he can simulate the other's position. Even by a combination of Internet and mobile cloud apps he can calculate the distance between the both sides and set out a variety of transportation solutions. This allows the user to have a better idea of the effect of experience.

\subsection{Development of cloud technology}

4G networks will make applications based cloud computing more attractive than it is now. If the $4 \mathrm{G}$ network transmission speed is 10 times faster then the cloud service for mobile users will become relatively more reliable, more practical and safe. While the development of cloud and $4 \mathrm{G}$ networks will promote the development of mobile / portable gaming, the development of navigation applications and a series of development including emergency response and telemedicine.

Cloud computing for the promotion of $4 \mathrm{G}$ services is not timely mature, but icing on the cake. As the application of next-generation network infrastructure technology, cloud computing just adapted to the enormous computing power requires of the $4 \mathrm{G}$ era, The "cloud" in the mobile terminal has become a trend of terminal development nowadays. Relative to the $3 \mathrm{G}$ technology, TD-LTE speed increase a few times which can greatly promote high-definition video on demand, high-speed downloads, online games and other recreational activities. This not only provides a better communication environment for the realization of cloud computing, als ostimulate user demand for cloud computing.

\section{CONCLUSION}

Relative to the $3 \mathrm{G}$ network, $4 \mathrm{G}$ wireless networks Introduced some new technologies, This makes the $4 \mathrm{G}$ wireless network is more high-end than the $3 \mathrm{G}$ network, and more technical. 4G wireless network make "cover the world" and "seamless service" as its basic goal. Through the efficient use of spectrum, dynamic allocation of broadband, security sublimation technology, transmission technology upgrades and other aspects $4 \mathrm{G}$ will achieve its goals. It was a full range of upgrades for the original $3 \mathrm{G}$ technology including network access, transport, security, service, communication, etc. 4G demand more on the transmission channel (broadband), but relying on intelligent technologies (spectrum allocation), it can use broadband more efficient to improve the transmission speed and the flow of information within the time unit. In addition, radio technology is the biggest feature of $4 \mathrm{G}$ wireless networks, through software to build virtual channels [8-10]. It is faster than the $3 \mathrm{G}$ network.

$4 \mathrm{G}$ networks will have a huge impact on cloud computing, e-commerce, wireless city and the wisdom of life related industries. It will promote industrial chain applications fully upgraded. $4 \mathrm{G}$ will bring a new era of mobile Internet, more applications will be fast complied on $4 \mathrm{G}$ channel. $4 \mathrm{G}$ has become an important carrier to promote the industry development of cloud computing, networking, mobile networking etc.

\section{CONFLICT OF INTEREST}

The authors confirm that this article content has no conflict of interest.

\section{ACKNOWLEDGEMENTS}

Declared none.

\section{REFERENCES}

[1] R. Ting, "Analysis and Comparison of 4G Wireless Networks and 3G Networks", Technology Forum, vol. 8, pp. 120-121, 2013.

[2] S. Trabelsi, "Noureddine Boudriga, Predictive Dynamic Uplink /Downlink Resource Reservation for Vertical Handoff Optimization in 4G Networks", International Journal of Business Data Communications and Networking (IJBDCN), vol.6, no.4, pp. 242 249, 2010. 
[3] G. Su, "Research on 4G mobile Communications Network Applications of TD-LTE standard", Information And Communications, vol.7, pp. 221-222, 2013.

[4] R. H. Bhuiyan, M. Ali, "A Double Meander PIFA with a Parasitic Metal Box for Wideband 4G Mobile Phones", In: Proceedings of the IEEE APSURSI, vol.3, pp. 1914-1917, 2011.

[5] M. A. Soliman, T. E. Taha, W. E. Swelam, and A. M. Gomaa, "A Wearable Dual-Band Dielectric Patch An- tenna for LTE and WLAN," Journal of Electromagnetic Analysis and Applications, vol.4, pp.305-309, 2012.

[6] A. N. Kulkarni, S. K. Sharman, "A band Antenna with MIMO Implementation for U4G LTE Wireless Devices," In: Proceedings of the IEEE APSURSI, vol.3, pp. 2215-2218, 2011.
[7] M. Han, and J. Choi, "MIMO antenna using a decoupling network for 4G USB dongle application," Microw Opt Technol Lett, vol.52, pp. 774-781, 2010.

[8] A. Elouadih, O.S. Ahmed, and M. M. Hassani, "Design and Simulation of a PIFA Antenna for the Use in 4G Mobile Telecommunications Networks," Int'l J. of Communications Network and System Sciences, vol. 06 , pp. 325-332, 2013.

[9] Z. Guennoun, and M. Jaloun, "Wireless Mobile Evolution to 4G Network," Wireless Sensor Network, vol.02, pp. 309-317, 2010.

[10] T. Liu, and H. Fang, "The key technology and development of 4G mobile communication system," Science \& Technology Information, vol.09, pp. 298-300.

Received: June 10, 2015

Revised: July 29,2015

Accepted: August 15, 2015

(c) QingXia et al.; Licensee Bentham Open.

This is an open access article licensed under the terms of the Creative Commons Attribution Non-Commercial License (http://creativecommons.org/licenses/by-nc/3.0/) which permits unrestricted, non-commercial use, distribution and reproduction in any medium, provided the work is properly cited. 